\title{
Human Emotion Recognition Using Heart Rate Variability Analysis with Spectral Bands Based on Respiration
}

\author{
María Teresa Valderas ${ }^{1,2}$, Juan Bolea ${ }^{1}$, Pablo Laguna ${ }^{1}$, Senior Member IEEE, \\ Montserrat Vallverdú ${ }^{2}$ and Raquel Bailón ${ }^{1}$
}

\begin{abstract}
The work presented in this paper aims at assessing human emotion recognition by means of the analysis of the heart rate variability (HRV) with varying spectral bands based on respiratory frequency (RF). Three specific emotional states are compared corresponding to calm-neutral state (Relax), positive elicitation (Joy) and negative elicitation (Fear). Standard HRV analysis in time and frequency domain is performed. In order to better characterize the HRV component related to respiratory sinus arrhythmia, the high frequency (HF) band is centered on RF. Results reveal that the power content in low band (PLF), the normalized power content in HF band (PHFn) and the sympathovagal ratio (LF/HF) can be suitable indices to distinguish Relax and Joy. Mean heart rate and RF are significantly different between Relax and Fear. Different HRV indices show significant differences between Joy and Fear, such as pNN50, PLF, PHFn and LF/HF. Statistical analysis of HRV indices with $\mathrm{HF}$ centered in the RF results in a lower $p$-value than the ones with a HF standard band.
\end{abstract}

\section{INTRODUCTION}

Both anatomically and functionally, autonomous nervous system (ANS) is composed of sympathetic and parasympathetic branches which exert an antagonistic regulation of target organs and tissues. These branches are named for acting in "sympathy" with emotions and both innervate the heart, specifically in the sinoatrial node which is in charge of neuromodulation enabling the corresponding neurotransmitters modulate its activity together which clearly responds to emotional states. Sympathetic hyperactivity in response to sexual or combative nature may cause extra systole or tachycardia. Parasympathetic hyperactivity in response to aversive emotions usually olfactory or visual origin can cause bradycardia or cardiac arrest [1], [2]. Heart rate variability (HRV) analysis is considered as a noninvasive technique for the assessment of the balance between these two main

* Research supported by Ministerio de Economía y Competitividad (MINECO), FEDER; under the project TIN2014-53567-R, by CIBER de Bioingeniería, Biomateriales y Nanomedicina through Instituto de Salud Carlos III, by Grupo Consolidado BSICoS from DGA (Aragón) and European Social Fund (EU), by University of Zaragoza by project UZ2014TEC-01, by CICYT grant TEC2013-44666-R and TEC2014-60337-R from the Spanish Government. The computation was performed by the ICTS "NANBIOSIS", more specifically by the High Performance Computing Unit of the CIBER in Bioengineering, Biomaterials \& Nanomedicne (CIBER$\mathrm{BBN}$ ) at the University of Zaragoza.

${ }^{1}$ MT. Valderas, J. Bolea, P. Laguna and R. Bailón are with the Biomedical Signal Interpretation and Computational Simulation (BSICoS), Aragón Institute for Engineering Research, IIS Aragón, University of Zaragoza, Spain, and CIBER de Bioingeniería, Biomateriales y Nanomedicina (CIBERBBN), María de Luna, 1, 50015 Zaragoza, Spain.

${ }^{2}$ MT. Valderas and M. Vallverdú are with the BarcelonaTech University and Biomedical Engineering Research Centre (CREB), Pau Gargallo, 5, 08028, Barcelona, Spain. maite.valderas @phdemotions . com branches of the ANS by its spectral analysis and it has been proposed for human emotion recognition [3], [4]. Standards of measurement, physiological interpretation and clinical use of HRV in resting conditions have been established, involving three different spectral components: a very low frequency (VLF) component in the range between 0 and $0.04 \mathrm{~Hz}$, a low frequency (LF) component between 0.04 and $0.15 \mathrm{~Hz}$, and a high frequency (HF) component between 0.15 and $0.4 \mathrm{~Hz}$ [5]. The power in the HF band is considered to be a measure of the parasympathetic activity, mainly due to respiratory sinus arrhythmia (RSA). The power in the LF band is considered to be a measure of sympathetic and parasympathetic activity on the heart, being its interpretation controversial, as e.g. when the respiratory frequency (RF) lies in the LF band. However, the spontaneous RF is not restricted to the band from 0.15 to $0.4 \mathrm{~Hz}$. For instance, the RF can be as low as $0.1 \mathrm{~Hz}$ during relaxation and as high as $0.7 \mathrm{~Hz}$ during intense exercise. In these situations the spectral analysis of HRV within the standard frequency bands would yield inaccurate estimates of the ANS activity. In order to avoid this effect, it has been proposed to center the HF band in the RF for an improved estimation of the sympathethic/parasympathetic activity [6]. The aim of this paper is to overcome this drawback in the context of HRV analysis during emotion elicitation using a HF band centered at RF, previously successfully tested in the context of stress testing [6], [7], [8]. This approach can be divided into three categories: (i) time domain analysis to obtain HRV statistical time indexes, (ii) frequency domain analysis based on nonparametric methods and (iii) same procedure as in the previous case but varying the HF spectral band based on respiration.

\section{METHODS}

\section{A. Induced emotion registration}

A database of electrocardiogram (ECG), respiration, blood pressure (BP), skin temperature (ST) and galvanic skin response (GSR) for twenty five subjects was recorded during induced emotion experiments at University of Zaragoza. The limb ECG leads I, II and III were recorded at 1 $\mathrm{kHz}$ and the respiratory signal at $125 \mathrm{~Hz}$. The Institutions Ethical Review Board approved all experimental procedures involving human subjects.

Four emotions (Joy, Fear, Anger and Sadness) were induced by videos in two different days, two sessions per day and two emotions each (one session for Joy and Fear and the other for Anger and Sadness). The order of the emotion 
TABLE I

Median (Q1|Q3) For HRV indices And RF for RelaX, Joy AND FEAR AND $p$-VAlues For the Studied Conditions: (1) Relax Vs. Joy, (2) RELAX VS. FEAR AND (3) JOY VS. FEAR.

\begin{tabular}{|c|c|c|c|c|c|c|}
\hline & \multicolumn{3}{|c|}{ Emotions } & \multicolumn{3}{|c|}{$p$-values } \\
\hline & Relax & Joy & Fear & (1) & (2) & (3) \\
\hline HRM (bpm) & $72.13(68.33 \mid 77.26)$ & $73.24(68.47 \mid 80.96)$ & $77.71(70.62 \mid 83.19)$ & n.s. & 0.0131 & n.s. \\
\hline SDNN (ms) & 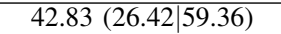 & $\begin{array}{l}48.95(31.73 \mid 58.07) \\
\end{array}$ & $\begin{array}{l}43.40(34.02 \mid 57.92) \\
\end{array}$ & n.s. & n.s. & n.s. \\
\hline SDSD (ms) & $25.95(15.57 \mid 35.82)$ & $28.47(17.21 \mid 39.03)$ & $25.32(17.39 \mid 30.48)$ & n.s. & n.s. & n.s. \\
\hline RMSSD (ms) & $\begin{array}{l}25.92(15.55 \\
35.74)\end{array}$ & $\begin{array}{l}28.44(17.19 \mid 38.98) \\
\end{array}$ & $\begin{array}{l}25.29(17.36 \\
30.43)\end{array}$ & n.s. & n.s. & n.s. \\
\hline pNN50 (\%) & $5.26(0.00 \mid 14.42)$ & $8.33(0.65 \mid 17.46)$ & $3.10(0.53 \mid 9.29)$ & n.s. & n.s. & 0.0098 \\
\hline PLF (adim) & $0.0010(0.0004 \mid 0.0015)$ & $0.0013(0.0006 \mid 0.0027)$ & $0.0010(0.0006 \mid 0.0019)$ & 0.0419 & n.s. & 0.0166 \\
\hline $\mathrm{PHF}^{a}$ (adim) & $0.0003(0.0001 \mid 0.0008)$ & $0.0005(0.0002 \mid 0.0008)$ & $\begin{array}{l}0.0004(0.0002 \mid 0.0006) \\
\end{array}$ & n.s. & n.s. & n.s. \\
\hline $\mathrm{PHFn}^{a}(\%)$ & \begin{tabular}{l|l|l|}
$31.40(19.26$ & $45.17)$ \\
\end{tabular} & $23.72(17.26 \mid 32.56)$ & \begin{tabular}{|l|l|}
$26.62(18.08$ & $40.14)$
\end{tabular} & 0.0009 & n.s. & 0.0004 \\
\hline $\mathrm{PLF} \mathrm{PHF}^{a}$ (adim) & $2.19(1.21 \mid 4.19)$ & $3.24(2.08 \mid 4.80)$ & $2.76(1.50 \mid 4.53)$ & 0.0052 & n.s. & 0.0006 \\
\hline $\mathrm{PHF}^{b}$ (adim) & $0.0002(0.0001 \mid 0.0008)$ & $0.0003(0.0001 \mid 0.0006)$ & $0.0003(0.0001 \mid 0.0005)$ & n.s. & n.s. & n.s. \\
\hline $\mathrm{PHFn}^{b}(\%)$ & $28.08(13.75 \mid 44.20)$ & $20.93(10.30 \mid 29.78)$ & $23.75(14.24 \mid 34.27)$ & 0.0006 & n.s. & 0.0012 \\
\hline${\mathrm{PLF} / \mathrm{PHF}^{b} \text { (adim) }}$ & $2.56(1.26 \mid 6.27)$ & $3.79(2.36 \mid 8.72)$ & $3.21(1.92 \mid 6.05)$ & 0.0004 & n.s. & 0.0004 \\
\hline $\mathrm{RF}(\mathrm{Hz})$ & $0.31(0.26 \mid 0.33)$ & $0.33(0.31 \mid 0.36)$ & $0.32(0.29 \mid 0.35)$ & n.s. & 0.0237 & n.s. \\
\hline
\end{tabular}

n.s. Stands for non-significant.

videos was randomized. Each emotion video was preceded by a relaxing video which served as physiological parameters baseline, and followed by another relaxing video to ensure that the subjects physiological parameters returned to the baseline condition.

Notwithstanding were recorded four basic emotions, only Joy and Fear were analyzed. These two basic emotions were selected following the emotion classification model of Lövheim, who proposed a direct relation between specific combinations of the levels of the signal substances dopamine, serotonin and noradrenaline and basic emotions represented in a three-dimensional model. Inside this coordinate system Joy and Fear were differentiated in a single dimension axis which was high and low level of serotonin, respectively [9].

\section{B. HRV representation}

First, beat occurrence times were estimated from the ECG by a wavelet-based ECG detector [10]. Then, ectopic beats, false and missed detections were identified and corrected before constructing the RR time series.

Besides the RR series, instantaneous heart rate (HR) was estimated from the beat occurrence times based on the integral pulse frequency modulation (IPFM) model, which takes into account the presence of ectopic beats [11].

A time-varying mean HR was computed by low pass filtering (cut-off frequency $0.03 \mathrm{~Hz}$ ) the instantaneous HR signal, and then subtracted from the instantaneous HR signal, yielding the HRV signal.

Finally, the modulating signal, which is assumed to carry the ANS information according to the IPFM model, was estimated dividing the HRV signal by the time-varying mean HR [12].

\section{HRV indices}

The first stage to analyze HRV is to obtain the standard temporal and spectral indices [5].

For time domain, heart rate mean (HRM), the standard deviation of consecutive normal beats (SDNN), the standard deviation of successive differences between adjacent normal beats (SDSD), the root mean square of successive differences between adjacent normal beats (RMSSD), and the proportion of beats that differ more than $50 \mathrm{~ms}$ (pNN50) were obtained from the revised RR time series.

Frequency domain indices were estimated from the modulating signal described in Section II-B using the Fourier transform. The power content in LF and HF bands (PLF and PHF), their normalized values (i.e. PHFn = $\mathrm{PHF} /(\mathrm{PLF}+\mathrm{PHF})$ ), and the sympathovagal ratio (LF/HF) were computed for two situations:

- Considering standard ranges [5].

- Considering the HF band centered in the RF [6].

A length of five minutes was considered for both sessions of Relax and Joy and for one session of Fear. The second session of Fear had an extension of 285 seconds due to a restriction in the duration of the video.

\section{HF band centered on respiratory frequency}

As stated above the standard definitions of the HF band could be inappropriate when the RF is not within to 0.15-0.4 $\mathrm{Hz}$.

The criterion used to overcome this limitation was:

- The HF band is centered in the RF $\left(\mathrm{HF}_{R F}\right)$ extracted from the respiratory signal, with the same bandwidth than the standard definition of HF, $0.25 \mathrm{~Hz}$.

- Two restrictions are applied to the previous definition: (1) The upper limit of the HF band must be limited by half the mean HR (Nyquist frequency), and (2) the lower limit of the HF band is defined from the upper limit of the standard LF band, $0.15 \mathrm{~Hz}$.

The RF was extracted by Welch periodogram from respiratory signal which was resampled at $4 \mathrm{~Hz}$.

\section{E. Statistical analysis}

Prior to the statistical analysis, normality distribution of all indices were evaluated by Lillie test resulting in most of them present nonnormal distribution. 

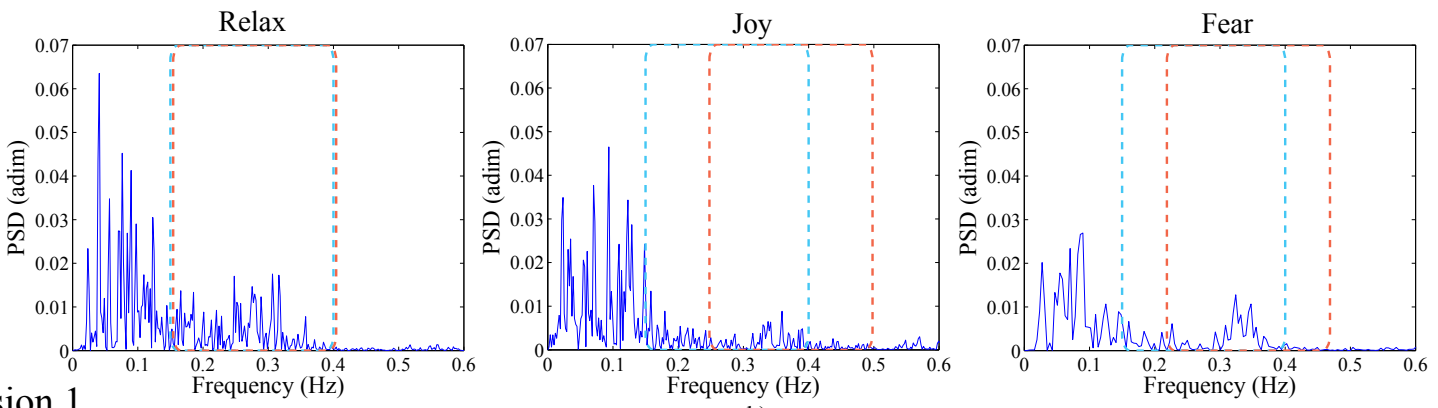

Session 1

a)

b)

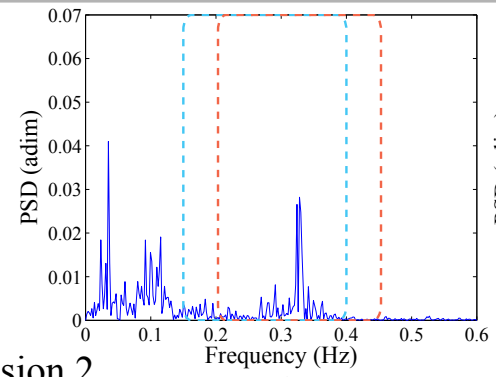

d)

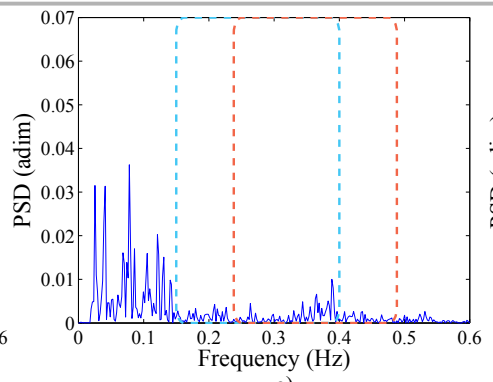

e)

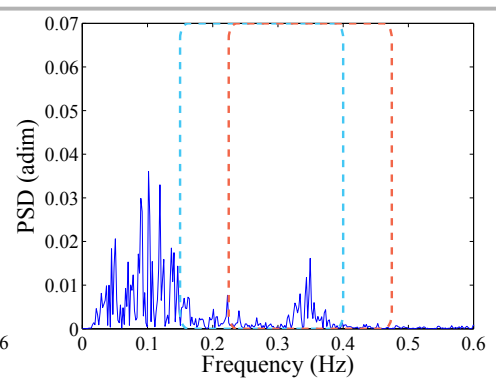

$---\mathrm{HF}$ Band $---\mathrm{HF}_{\mathrm{RF}}$ Band

f)

Fig. 1. Power Spectral Density (PSD) of the HRV corresponding to subject 18 for Relax (a and d), Joy (b and e) and Fear (c and f) for both sessions.

Therefore, statistical analysis was done by a MannWhitney U-test to evaluate statistical significant differences for all conditions: (1) Relax vs. Joy, (2) Relax vs. Fear and (3) Joy vs. Fear. A $p$-value $<0.05$ was considered as statistically significant.

\section{RESULTS}

The HRV indices are shown in Tab. I in terms of median and interquartile ranges as first (Q1) and third (Q3) quartile, median $(\mathrm{Q} 1 \mid \mathrm{Q} 3)$, calculated for standard statistical analysis in time domain, frequency domain with standard HF range and with HF based on RF and the RF for Relax, Joy and Fear. $p$-values obtained from the Mann-Whitney U-test are shown also in Tab. I for the three conditions studied: (1) Relax vs. Joy, (2) Relax vs. Fear and (3) Joy vs. Fear.

HRV spectrum corresponding to Relax, Joy and Fear is illustrated in Fig. 1 a), b), c) for session 1 and d), e), f) for session 2, to observe power content differences. The HF standard band (HF Band) and the HF band centered in the respiratory frequency $\left(H F_{R F}\right.$ Band) are remarked inside these six pictures.

All pictures are from the same subject measured into both sessions but different days. This consideration was taken in account to compare the power content but avoiding extra environmental factors in the analysis of the behavior of the signal.

\section{DISCUSSION}

The analysis of the HRV indices revealed statistical significant differences among emotions: (1) Relax vs. Joy, (2) Relax vs. Fear and (3) Joy vs. Fear. Therefore, as it was shown in Tab. I, it was possible to differentiate human feelings during emotion elicitation using HRV analysis.
It was observed that spectral indices (PLF, PHFn and $\mathrm{LF} / \mathrm{HF}$ ) of HRV could be suitable indices to distinguish between Relax and Joy. Results suggest a more sympathetic ANS balance during Joy than during Relax, as showed Fig. $1 \mathrm{a}), \mathrm{b}), \mathrm{d})$ and e).

The pNN50 index showed statistical differences between Joy and Fear as well as spectral indices as PLF, PHFn and $\mathrm{LF} / \mathrm{HF}$.

Note that many differences between Joy and Fear were similar to those between Joy and Relax, and that in this experiment the indices which were statistically different between Relax and Fear were HRM and RF. The absence of statistical significance in ANS related indices may be due to the degree in which the Fear emotion can be induced by a video. In fact, in the comparison between Joy and Fear, a more sympathetic ANS balance is observed in Joy than in Fear (Fig. 1 b), c), e) and f)).

Being more strict in the statistical analysis by considering the Bonferroni correction only PLF lost statistical significance in the comparison Relax vs. Joy and RF in the comparison Relax vs. Fear.

It should be underlined that no single index showed statistical differences among the three studied conditions.

Since spectral analysis of HRV was performed on the modulating signal, which represents the variations of HRV not due to variations in mean HR [11], we hypothesized that changes measured by HRV reflect changes in ANS rather than changes in mean HR. The statistical analysis of the indices derived from HF centered in the RF resulted in a lower $p$-value than the ones from HF standard band supporting the proposal (Tab. I).

In the whole study the standard LF band was not modified. 
Those cases where the RF was found lower than $0.20 \mathrm{~Hz}$, the bandwidth of the HF band decreased dramatically so the estimation of the sympathetic and parasympathetic activity was not a reliable measurement. This situation occurred mostly during Relax conditions since it was the condition with a lower RF, as show in Tab. I. Due to this limitation, respiratory frequencies below $0.20 \mathrm{~Hz}$ were not considered for this study.

Comparing these results with the literature, HRV analysis is a suitable technique for the assessment of the balance of ANS and therefore for human emotion recognition states as stress [6], [7], [8], panic [13], [14], anxiety and depression [3] among others, even for healthy or illness people, with significant results in the spectral domain for LF, HF and LF/HF.

The same analysis could be extended by considering the emotions not implemented in this paper (Anger and Sadness) and the physiological data (BP, ST and GSR) simultaneous registered with the ECG and respiration during this experiment, with the finality to extract the maximum information of our framework.

As a future line, the inclusion of other HRV indices such as nonlinear could improve the description of emotions in terms of ANS activity with the aim of being able to classify them accurately.

\section{CONCLUSIONS}

In this work HRV has been analyzed during three emotional states: Relax, Joy and Fear. Temporal and frequency indices of HRV have been studied, including a high frequency band centered on respiratory frequency. Statistical significance differences were found for spectral domain indices distinguishing Relax vs. Joy, for time domain indices and the respiratory frequency for Relax and Fear, and for spectral and temporal domains for Joy and Fear. Strong statistical differences were obtained for the analysis with high frequency centered in the respiratory frequency pointing out the necessity of considering a specific high frequency band on each case in order not to lose relevant information.

\section{REFERENCES}

[1] M.J. Turlough Fitzgerald, G. Gruener, and E. Mtui. Neuroanatomía clínica y neurociencia. Elsevier Saunders, pages 149-154, 2012.

[2] A. Despopoulos and S. Silbernagl. Color atlas of physiology. Basic Sciences(Thieme), pages 192-195, 2003.

[3] D.S. Quintana, A.J. Guastella, T. Outhred, I.B. Hickie, and A.H. Kemp. Heart rate variability is associated with emotion recognition: direct evidence for a relationship between the autonomic nervous system and social cognition. Int. J. of Psychophysiol, 86(2):168-172, 2012.

[4] G. Chanel, J.M. Kierkels, M. Soleymani, and T. Pun. Short-term emotion assessment in a recall paradigm. Int. J. Human-Computer Studies, 67(8):607-627, 2009.

[5] Task Force of ESC and NASPE. Heart rate variability. standards of measurement, physiological interpretation, and clinical use. Eur. Heart J., 17:354-381, 1996.

[6] R. Bailón, P. Laguna, L. Mainardi, and L. Sörnmo. Analysis of heart rate variability using time-varying frequency bands based on respiratory frequency. In IEEE EMBS International Conference on Engineering in Medicine and Biology Society, 29th International Conference on, pages 6674-6677, 2007.

[7] R. Bailón, L. Mainardi, and P. Laguna. Time-frequency analysis of heart rate variability during stress testing using a priori information of respiratory frequency. In Computers in Cardiology, pages 169-172, 2006

[8] R. Bailón, L. Mainardi, M. Orini, L. Sörnmo, and P. Laguna. Analysis of heart rate variability during stress testing using respiratory information. Biomed. Signal Process. Control, 5(4):299-310, 2010.

[9] H. Lövheim. A new three-dimensional model for emotions and monoamine neurotransmitters. Med. Hypotheses, 78(2):341-8, 2012.

[10] J. P. Martínez, R. Almeida, S. Olmos, A. P. Rocha, and P. Laguna Wavelet-based ECG delineator: evaluation on standard databases. IEEE Trans. Biomed. Eng., 51:570-581, 2004.

[11] J. Mateo and P. Laguna. Analysis of heart rate variability in the presence of ectopic beats using the heart timing signal. IEEE Trans. Biomed. Eng., 50(3):334-343, 2003.

[12] R. Bailón, G. Laouini, C. Grao, M. Orini, and P. Laguna. The integral pulse frequency modulation model with time-varying threshold: Application to heart rate variability analysis during exercise stress testing. IEEE Trans. Biomed. Eng., 58(3):642-652, 2011.

[13] A. Rantanen, S.J. Laukka, M. Lehtihalmes, and T. Seppaänen. Heart rate variability reflecting from oral reports on negative experience. Procedia Soc. Behav. Sci., 5:483-487, 2010.

[14] J.M. Martinez, A. Garakani, C.J. Aaronson, and J.M. Gorman. Heart rate and respiratory response to doxapram in patients with panic disorder. Psychiat. Res., page In press, 2015. 